\title{
IDENTIFICAÇÃO DO PACIENTE EM NEONATOLOGIA PARA ASSISTÊNCIA SEGURA
}

\author{
Adriana Pereira Trindade de Souza Gomes ${ }^{1}$, Danielle Lemos Querido², Gloria Regina Gomes da Silva ${ }^{3}$, Luana \\ Ferreira de Almeida ${ }^{4}$, Ronilson Gonçalves Rocha ${ }^{5}$
}

\begin{abstract}
RESUMO: Estudo transversal, observacional, documental, abordagem quantitativa, realizado numa Unidade Neonatal de um Hospital Universitário do Rio de Janeiro, no período de maio e agosto de 2016. Analisou a identificação de pacientes neonatos por meio de pulseira. Foram realizadas 200 observações; constatou-se que em 155 (77,5\%), os neonatos apresentavam pulseiras de identificação. Quanto à localização, 91 (58,7\%) delas estavam em tornozelos ou punhos; quanto à legibilidade dos descritores, 76 (83,5\%) apresentavam-se em conformidade. Em $46(89,3 \%)$ observações não houve checagem da pulseira antes da entrega do neonato ao familiar, assim como não houve a checagem antes da realização de procedimentos em $45(88,3 \%)$ casos. Não houve registros em prontuários quanto à presença da pulseira em 174 (87\%) casos, e localização em 179 (89,5\%). Concluiu-se que a unidade investigada apresenta falhas relacionadas ao processo de identificação de seus pacientes, indicando que a implementação de estratégias para assistência segura é necessária e urgente.
\end{abstract}

DESCRITORES: Neonatologia; Segurança do paciente; Gerenciamento de risco; Enfermagem.

\section{THE IMPORTANCE OF NEWBORN IDENTIFICATION TO THE DELIVERY OF SAFE PATIENT CARE}

ABSTRACT: A cross-sectional, observational and documentary study with a quantitative approach, performed at a Neonatal Unit of a University Hospital of Rio de Janeiro, from May to August 2016. Identification of neonatal patients through the use of bands was assessed. For this purpose, 200 observations were made. It was found that 155 (77.5\%) newborn infants had identification bands. As for the place, $91(58.7 \%)$ of the bands were around the ankles or wrists of the infants. Regarding the legibility of the information on the ID bands, this requirement was met in the ID bands of $76(83.5 \%)$ newborn infants. In $46(89.3 \%)$ observations, the ID band was not checked before the newborn was handed to a parent, nor was it checked prior to procedures in 45 (88.3\%) of the cases. The patients' medical records included no information regarding identification bands in 174 (87\%) cases, and its place in 179 (89.5\%) of the cases. It was concluded that the referred neonatal unit failed to provide appropriate identification of the patients, and the implementation of strategies for improving safe care is urgently needed.

DESCRIPTORS: Neonatology; Patient safety; Risk management; Nursing.

\section{IDENTIFICACIÓN DEL PACIENTE EN NEONATOLOGÍA PARA ASISTENCIA SEGURA}

RESUMEN: Estudio transversal, observacional, documental, de abordaje cuantitativo, realizado en una Unidad Neonatal de un Hospital Universitario de Rio de Janeiro, en el periodo de mayo y agosto de 2016. Se analizó la identificación de pacientes neonatos por medio de pulsera. Se realizaron 200 observaciones, donde se constató que en $155(77,5 \%)$ de los casos, los neonatos presentaban pulseras de identificación. Acerca de la localización, 91 (58,7\%) de ellas estaban en tobillos o muñecas; en cuanto a la legibilidad de los descriptores, $76(83,5 \%)$ se presentaban de acuerdo con el deseable. No hubo chequeo de la pulsera en 46 (89,3\%) observaciones antes de entregar el neonato al familiar. Tampoco hubo chequeo antes de la realización de procedimientos en 45 (88,3\%) casos. No hubo registros en prontuarios acerca de la presencia de la pulsera en 174 (87\%) casos, y localización en 179 (89,5\%). Se concluye que la unidad investigada apunta fallas referentes al proceso de identificación de sus pacientes, indicando que la implementación de estrategias para asistencia segura es necesaria y urgente.

DESCRIPTORES: Neonatología; Seguridad del paciente; Gestión de riesgo; Enfermería.

${ }^{1}$ Enfermeira. Especialista em Enfermagem Neonatal. Universidade do Estado do Rio de Janeiro. Rio de Janeiro, RJ, Brasil. ${ }^{2}$ Enfermeira. Mestre em Enfermagem. Enfermeira da Unidade Neonatal do Hospital Universitário Pedro Ernesto da Universidade do Estado do Rio de Janeiro. Rio de Janeiro, RJ, Brasil.

${ }^{3}$ Enfermeira. Mestre em Enfermagem. Chefe da Unidade Neonatal do Hospital Universitário Pedro Ernesto da Universidade do Estado do Rio de Janeiro. Rio de Janeiro, RJ, Brasil.

${ }^{4}$ Enfermeira. Doutora em Educação em Ciências e Saúde. Docente de Enfermagem da Faculdade de Enfermagem da Universidade do Estado do Rio de Janeiro. Rio de Janeiro, RJ, Brasil.

${ }^{5}$ Enfermeiro. Doutor em Enfermagem. Docente de Enfermagem da Faculdade de Enfermagem da Universidade do Estado do Rio de Janeiro. Rio de Janeiro, RJ, Brasil.

Autor Correspondente:

Adriana Pereira Trindade de Souza Gomes

Recebido: 29/11/2016

Universidade do Estado do Rio de Janeiro

Finalizado: 06/07/2017

R. Alcobaça, 422 - 21640-002 - Rio de Janeiro, RJ, Brasil

E-mail: adrianaptsgomes@gmail.com 


\section{INTRODUÇÃO}

A população de recém-nascido ( $R N$ ) internado em unidades hospitalares está exposta a inúmeros eventos adversos devido à ocorrência de erros no processo de identificação do paciente. Frequentemente, as imprensas brasileira e internacional noticiam casos como a troca de bebês no ambiente hospitalar, gerando sérias consequências psicossociais para as famílias envolvidas. Inúmeras vezes também são divulgadas circunstâncias em que medicamentos e procedimentos são realizados, por engano, em outros pacientes, geralmente por erros de identificação.

A forma de se identificar um paciente pediátrico ainda é preocupante, uma vez que existe grande escassez de estudos sobre o tema, especialmente por ser uma área específica e com várias particularidades, sendo importante o desenvolvimento de novas pesquisas que abordem a segurança do paciente ${ }^{(1)}$.

Esse tema está intrinsecamente relacionado com a qualidade da assistência à saúde, e vem sendo amplamente discutido por serviços e entidades de classe, bem como por órgãos governamentais ${ }^{(2)}$. A simples presença do paciente em unidade hospitalar representa inúmeros riscos à sua saúde ${ }^{(3)}$ e, quando se trata de pacientes pediátricos, os riscos são ainda maiores, pois correspondem a uma população com características físicas e morfológicas específicas, portanto, diferenciada dos adultos.

No contexto da atenção à saúde de neonatos de muito baixo peso ou prematuros, os riscos são ainda maiores, pois são manipulados por diversos profissionais e expostos a um número muito maior de procedimentos diagnósticos e tratamento, além de permanecerem por tempo mais prolongado no ambiente hospitalar. Esses fatores aumentam significativamente as chances de sofrerem as consequências de um erro ${ }^{(4)}$. É a partir da correta identificação do paciente que se torna possível garantir assistência segura ao paciente internado e ainda resultados mais efetivos ${ }^{(1)}$.

Compreendendo a importância do estabelecimento de medidas de segurança do paciente nas unidades de saúde do País, no ano de 2013, entrou em vigor a Portaria do Ministério da Saúde no 529, a qual instituiu o Programa Nacional de Segurança do Paciente (PNSP), cujo objetivo foi contribuir para a qualificação do cuidado em todos os estabelecimentos de saúde no Brasil( ${ }^{(5)}$.

Frente ao contexto apresentado, definiu-se como objeto de investigação deste estudo a identificação do paciente neonato por meio de pulseiras. Buscou-se analisar se os RN de uma Unidade Neonatal de um Hospital Universitário do Rio de Janeiro são corretamente identificados como preconiza o PNSP.

\section{- MÉTODO}

Trata-se de estudo transversal, observacional, documental, com abordagem quantitativa. Para o seu desenvolvimento, foram realizadas observações e registros sobre a população estudada, em um período de tempo delimitado ${ }^{(6-7)} \mathrm{com}$ vistas à obtenção de informações sobre o objeto investigado. $\mathrm{O}$ estudo também utilizou fontes específicas para obtenção de dados complementares (prontuários), os quais subsidiaram e complementaram, no momento de ocorrência dos fatos ou posteriormente, informações relevantes para a pesquisa ${ }^{(8)}$.

O estudo foi realizado na Unidade Neonatal de um Hospital Universitário, localizado no município do Rio de Janeiro. Tal unidade possui 24 leitos distribuídos da seguinte forma: oito leitos para cuidados semi-intensivos e 16 leitos para cuidados intensivos. Os RN recebem cuidados de equipe multiprofissional, que inclui residentes, docentes e acadêmicos de graduação e pós-graduação de diversas áreas da saúde.

Os neonatos atendidos nesta unidade são, em geral, portadores de patologias, como asfixia perinatal, prematuridade extrema, baixo peso ao nascer, insuficiência respiratória e/ou cardíaca, insuficiência hepática ou renal, e síndromes genéticas. Os pais dos $\mathrm{RN}$ recebem autorização da unidade para o acompanhamento permanente dos seus filhos, durante toda a internação, caso desejem.

Foram incluídos na pesquisa todos os RN internados na UTI neonatal no período da coleta de dados. Excluídos aqueles que se encontravam fora da unidade por algum motivo, por exemplo, realizando 
procedimentos como ultrassonografia transfontanela, ecocardiograma e cirurgias.

A coleta de dados foi realizada por meio de dois formulários. Um correspondia ao instrumento de observação do RN com informações sobre presença, localização, integridade e legibilidade da pulseira, presença de identificadores, definidos pelo Ministério da Saúde ${ }^{(9)}$ e itens voltados para o risco de lesões de pele provocadas pelas pulseiras ao neonato. Além disso, no primeiro formulário era registrada a atitude dos profissionais quanto à checagem da pulseira, antes da entrega do RN à mãe e/ ou a outro familiar e antes de realização de procedimentos.

O outro instrumento utilizado foi um formulário de avaliação de registros de enfermagem em prontuários, o qual possuía perguntas referentes ao registro diário sobre: presença, localização, integridade, colocação e legibilidade da pulseira, além de registros da necessidade de troca ou solicitação de nova pulseira.

A coleta de dados foi realizada em 25 dias em horários aleatórios, no período diurno, entre os meses de maio a agosto de 2016. Totalizaram-se 200 observações, obtendo-se uma média de oito observações diárias.

Os dados foram tabulados e demonstrados por variáveis quantitativas, analisados por meio de estatística descritiva, com uso de frequência absoluta (n) e relativa (\%) com exibição em tabelas.

Esta pesquisa seguiu os preceitos éticos apostos na Resolução do Conselho Nacional de Saúde $\mathrm{n}^{\circ}$ 466, de 12 de dezembro de 2012 e foi submetida ao Comitê de Ética em Pesquisa (CEP) da instituição onde foi desenvolvida ${ }^{(10)}$. Os responsáveis legais de todos os RN envolvidos receberam o TCLE, bem como os profissionais que concordaram em participar da pesquisa, autorizando a observação das suas ações e práticas profissionais no que se refere à checagem das pulseiras. Foi garantido o anonimato e sigilo das informações, conforme exigências da legislação vigente. Este estudo foi aprovado em 30/03/2016 pelo CEP institucional por meio do parecer $\mathrm{n}^{\circ} 1.436 .450$.

\section{RESULTADOS}

Durante a coleta de dados foram realizadas 200 observações. A cada dia de observação um neonato poderia ser alvo de novas observações, se permanecesse internado na Unidade Neonatal, no período de coleta de dados da pesquisa.

A repetição das observações sobre os mesmos participantes não representou vieses para os resultados da pesquisa, uma vez que o paciente identificado em determinado dia poderia não estar presente em outro dia, posto que as pulseiras, frequentemente, são retiradas para realização de procedimento, e podem não ser recolocadas. Da mesma forma, RN que não estavam identificados em certo dia, podiam estar com a pulseira de identificação em outro momento.

Na descrição dos resultados, inicialmente, são apresentados dados sobre a observação da pulseira de identificação no RN e, posteriormente, são descritos os dados relacionados aos registros de enfermagem contidos nos prontuários e referentes à pulseira de identificação.

\section{Dados relacionados à pulseira de identificação no $\mathbf{R N}$}

Entre as 200 observações realizadas, verificou-se que em 155 (77,5\%) os RN possuíam uma ou duas pulseiras de identificação, cuja procedência era do Centro Obstétrico (CO) ou do setor de internação. Cada pulseira encontrava-se localizada em um dos membros superiores ou inferiores do neonato ou fixada no leito (Tabela 1).

Tabela 1 - Pulseira de identificação nos Neonatos. Rio de Janeiro, RJ, Brasil, 2016

\begin{tabular}{|c|c|c|c|c|c|c|}
\hline & \multicolumn{2}{|c|}{ Sim } & \multicolumn{2}{|c|}{ Não } & \multicolumn{2}{|c|}{ Total } \\
\hline & $\mathbf{N}$ & $\%$ & $\mathbf{N}$ & $\%$ & $\mathbf{N}$ & $\%$ \\
\hline Pulseira de identificação no neonato & 155 & 77,5 & 45 & 25,5 & 200 & 100 \\
\hline
\end{tabular}


Nas 155 observações em que os neonatos possuíam alguma pulseira, foi verificado que 91 (58,7\%) delas encontravam-se localizadas nos membros da criança (tornozelos ou punhos), e 64 restantes $(41,3 \%)$ estavam fixadas no leito (Tabela 2$)$.

Tabela 2 - Localização da pulseira de identificação nos Neonatos. Rio de Janeiro, RJ, Brasil, 2016

\begin{tabular}{lccccccl} 
& \multicolumn{2}{c}{$\begin{array}{c}\text { No neonato } \\
\text { (tornozelos ou punhos) }\end{array}$} & \multicolumn{2}{c}{ No leito } & \multicolumn{2}{c}{ Total } \\
\cline { 2 - 7 } & $\mathbf{N}$ & $\%$ & $\mathbf{N}$ & $\%$ & $\mathbf{N}$ & $\%$ \\
\hline Localização da pulseira de identificação & 91 & 58,7 & 64 & 41,3 & 155 & 100
\end{tabular}

O Procedimento Operacional Padrão (POP) da instituição orienta que todos os neonatos internados na Unidade Neonatal estejam identificados com as duas pulseiras de identificação: aquela proveniente do setor de internação, e aquela proveniente do Centro Obstétrico, ambas fixadas no tornozelo e/ou punho do paciente.

Verificou-se que dentre 91 observações em que os neonatos possuíam pulseiras de identificação, fixadas em membros superiores e inferiores, 29 (31,9\%) RN possuíam as duas pulseiras, concordando com a recomendação aposta no documento institucional para a identificação correta do RN (Tabela 3).

Tabela 3 - Tipos de pulseira de identificação nos Neonatos. Rio de Janeiro, RJ, Brasil, 2016

\begin{tabular}{lll} 
Tipos de pulseira & N & \% \\
\hline Somente pulseiras do CO & 35 & 38,4 \\
\hline Somente pulseiras do setor de internação & 27 & 29,7 \\
\hline Pulseiras do Centro Obstétrico + setor de internação & 29 & 31.9 \\
\hline Total & 91 & 100
\end{tabular}

Dentre as 91 pulseiras presentes em um dos membros dos RN, identificou-se que 76 (83,5\%) delas estavam com descritores legíveis e não foi observado qualquer tipo de lesão ou garroteamento do membro em que se localizava a pulseira no neonato.

Não foi possível verificar a checagem da pulseira pelo profissional de enfermagem em 40 (21,9\%) observações. Isto ocorreu devido à realização simultânea de procedimentos em neonatos diferentes, no momento da observação. Das observações possíveis (51), verificou-se que não houve checagem da pulseira de identificação antes da entrega do neonato à mãe ou a outro familiar ( $n=46 / 89,3 \%$, bem como antes da realização dos procedimentos ( $n=45 / 88,3 \%)$.

\section{Dados da pulseira de identificação registrados em prontuário pela enfermagem}

Das 200 avaliações realizadas nos prontuários dos neonatos, observou-se que em 174 (87\%) não houve registro diário da presença da pulseira pelos profissionais de enfermagem. Quanto à localização da pulseira de identificação, $179(89,5 \%)$ dos registros não possuíam este tipo de anotação. Em relação à integridade da pulseira, em 195 (97,5\%) este tipo de relato não foi encontrado. Também não havia qualquer referência acerca da legibilidade dos descritores em 197 (98,5\%) dos registros nos prontuários (Tabela 4). 
Tabela 4 - Registro acerca da pulseira de identificação, nos prontuários dos Neonatos. Rio de Janeiro, RJ, Brasil, 2016

\begin{tabular}{lcccccc} 
& \multicolumn{2}{c}{ Sim } & \multicolumn{2}{c}{ Não } & \multicolumn{2}{c}{ Total } \\
\cline { 2 - 7 } & $\mathbf{N}$ & $\mathbf{\%}$ & $\mathbf{N}$ & $\mathbf{\%}$ & $\mathbf{N}$ & $\mathbf{\%}$ \\
\hline Presença & 26 & 13 & 174 & 87 & 200 & 100 \\
\hline Localização & 21 & 10,5 & 179 & 89,5 & 200 & 100 \\
\hline Integridade & 5 & 2,5 & 195 & 97,5 & 200 & 100 \\
\hline Legibilidade dos descritores & 3 & 1,5 & 197 & 98,5 & 200 & 100
\end{tabular}

Não foi identificado registro por profissionais de enfermagem sobre a necessidade de troca da pulseira em 196 (98\%) avaliações. Para os casos em que houve registro sobre necessidade de troca, havia também a solicitação de uma nova pulseira. Não havia, entretanto, anotações sobre a colocação da nova pulseira em $163(81,5 \%)$ registros avaliados, como descrito na Tabela 5.

Tabela 5 - Registro sobre a necessidade de troca, solicitação e colocação de nova pulseira. Rio de Janeiro, RJ, Brasil, 2016

\begin{tabular}{lcccccc} 
& \multicolumn{2}{c}{ Sim } & \multicolumn{2}{c}{ Não } & \multicolumn{2}{c}{ Total } \\
\cline { 2 - 7 } & $\mathbf{N}$ & $\mathbf{\%}$ & $\mathbf{N}$ & $\mathbf{\%}$ & $\mathbf{N}$ & $\mathbf{\%}$ \\
\hline Necessidade de troca & 4 & 2 & 196 & 98 & 200 & 100 \\
\hline Solicitação de nova pulseira & 4 & 2 & 196 & 98 & 200 & 100 \\
\hline Colocação da pulseira & 37 & 18,5 & 163 & 81,5 & 200 & 100
\end{tabular}

\section{- DISCUSSÃO}

Nesta pesquisa, constatou-se que em $77,5 \%$ das observações os $\mathrm{RN}$ possuíam alguma pulseira de identificação, e que em $58,7 \%$ destas observações as pulseiras estavam fixadas nos membros do neonato, como recomenda o Ministério da Saúde ${ }^{(9)}$. Das observações em que os RN possuíam pulseira de identificação localizada em membro (tornozelo ou punho), 29 (31,9\%) estavam com duas pulseiras, conforme recomenda instituição hospitalar investigada.

Deve-se considerar que a identificação é a primeira meta internacional de segurança do paciente ${ }^{(11)}$. Além de simples, a identificação por meio das pulseiras é um método eficaz, financeiramente acessível e que pode evitar falha grave/eventos adversos na prestação da assistência de enfermagem.

A ausência de identificação do paciente pode acarretar eventos adversos graves, tais como erros de administração de medicamentos ou hemoderivados, procedimentos realizados em pacientes trocados e/ou em locais inadequados, troca de bebês, entre outros ${ }^{(12)}$.

Uma possibilidade para a baixa adesão a essa medida de segurança pode ser o fato de alguns RN permanecerem por muito tempo internados. Tal situação pode gerar, nos profissionais da enfermagem, a falsa sensação do saber identificar o RN em qualquer momento, negligenciando, assim, a presença e checagem da pulseira de identificação.

De acordo com a OMS, entre novembro de 2003 e julho de 2005, o United Kingdom National Patient Safety Agency citou 236 incidentes relacionados à ausência ou a erro na identificação da pulseira(13). Por outro lado, em pesquisa realizada numa Unidade Neonatal com 540 observações, envolvendo o protocolo de identificação do RN, $82,2 \%$ estavam em conformidade ${ }^{(14)}$.

Na presente pesquisa, a partir das 200 observações realizadas, constatou-se que em 155 (77,5\%) os neonatos apresentavam pulseiras de identificação. Quanto à localização verificou-se que 91 
$(58,7 \%)$ estavam em tornozelos ou punhos, e quanto à legibilidade dos seus descritores $76(83,5 \%)$ apresentavam-se em conformidade. Constatou-se, entretanto, que em 46 (89,3\%) observações não houve a checagem da pulseira antes da entrega do neonato ao familiar, assim como não houve a checagem antes da realização de procedimentos em $45(88,3 \%)$ casos. Também não houve registros em prontuários quanto à presença da pulseira em 174 (87\%) casos, e localização em 179 (89,5\%). Desta maneira, percebeu-se que os neonatos, na unidade investigada, estavam expostos a grande risco de ocorrência de eventos adversos.

A assistência hospitalar décadas atrás era considerada mais simples, menos efetiva e menos segura; atualmente, apresenta-se mais efetiva, complexa e com maior risco ${ }^{(15)}$. É necessário que os serviços de saúde, bem como os profissionais, acompanhem esta evolução para que seja possível a oferta de saúde eficiente e segura para a população.

A semelhança entre os neonatos, dadas as suas aparências físicas, pode acarretar falhas na identificação antes de algum procedimento ${ }^{(16)}$. Outro fator importante que pode induzir ao erro é o fato destes pacientes ainda não se comunicarem verbalmente com a equipe, dificultando a confirmação de sua identificação, como se procede com pacientes adultos. Importante destacar que a execução de procedimentos em pacientes errados é uma possibilidade real e que nenhuma instituição ou profissional de saúde está livre deste risco ${ }^{(3)}$.

No ano de 2012, pesquisa realizada em uma maternidade do nordeste do Brasil mostrou que, dentre os $218 \mathrm{RN}$ que participaram do estudo, 183 (84\%) apresentaram algum tipo de evento adverso. O mesmo estudo revelou que $76 \%$ dos casos de eventos adversos resultaram em de danos temporários e/ou prolongamento do tempo de internação hospitalar. A referida pesquisa apontou que $87 \%$ dos eventos adversos estudados eram preveníveis ${ }^{(17)}$, considerando-se o que é preconizado pela literatura vigente sobre a identificação do paciente para uma assistência segura.

Ressalta-se, na presente pesquisa, o fato da maior parte das pulseiras presentes nos RN serem provenientes do Centro Obstétrico. Tal situação, possivelmente, seja justificada devido ao fato de o Centro Obstétrico ter rotina estabelecida de promover a identificação após o nascimento de todos os $\mathrm{RN}$.

Quanto à legibilidade das pulseiras, observou-se que 76 (83,5\%) encontravam-se adequadas. Estudo realizado no ano de 2012 avaliou a mesma variável em pacientes neonatos; verificou-se que 93,1\% das pulseiras encontravam-se adequadas, semelhante ao resultado do estudo apresentado ${ }^{(14)}$.

Algumas causas podem ser responsáveis pelo bom índice de legibilidade da pulseira: neonatos, especialmente os prematuros, possuem pouca movimentação, o que pode diminuir o atrito e aumentar a durabilidade da pulseira. Também o fato de a maioria dos neonatos estar em protocolo de mínimo manuseio, diminuindo o atrito.

Não foi identificada, durante a pesquisa, lesão relacionada ao uso da pulseira. Observou-se que a Unidade Neonatal investigada contava com uma equipe de enfermagem numericamente expressiva, $o$ que possibilitou provavelmente cuidado mais direcionado e atento.

O protocolo de identificação correta do paciente da ANVISA recomenda que a informação contida na pulseira deve ser checada todas as vezes que o recém-nascido for entregue à mãe ou responsável legal ${ }^{(10)}$. No entanto, em 58 casos $(89,3 \%)$ a pulseira não foi checada antes da entrega do neonato para o responsável legal ou familiar. Tal fato expõe tanto a criança quanto a família ao risco desnecessário de graves problemas sociais e emocionais.

Durante as observações, em 68 momentos $(88,3 \%)$ a pulseira não foi checada antes da realização dos procedimentos. Isto é preocupante, visto que na maioria dos casos os RN foram expostos a grande risco de eventos adversos, tais como administração de medicamentos errados, cateterismos epicutâneo desnecessários, encaminhamento do paciente errado para cirurgia, entre outras situações.

Em se tratando de erros relacionados à identificação do paciente, uma pesquisa realizada no estado de São Paulo analisou 74 erros relacionados a medicamentos ocorridos em uma unidade de clínica médica. Demonstrou que 2,7\% dos erros de administração de medicamentos ocorreram por não checagem da identificação do paciente antes da administração do fármaco ${ }^{(18)}$. Outro estudo, 
realizado em uma unidade pediátrica de um Hospital Escola de Santa Catarina, demonstrou que de 223 procedimentos de administração de medicamentos e fluidos observados, em 81 (36,32\%) não houve nenhum tipo de identificação do paciente ${ }^{(3)}$.

Endossando a importância da correta identificação do paciente, todos os profissionais devem participar ativamente deste processo, incluindo a pré-alta, transferência, ou antes da realização de qualquer procedimento ${ }^{(12)}$. No entanto, cotidianamente, a verificação de pulseiras do paciente por vezes é omitida pelos profissionais de saúde, principalmente naqueles que permanecem em longo período de internação ${ }^{(2)}$.

Nesse sentido, torna-se cada vez mais importante orientar os profissionais quanto à necessidade de seguimento aos protocolos de segurança do paciente. A adequada identificação do paciente possibilita assegurar assistência com menos risco de erros e danos aos mesmos ${ }^{(1)}$. Como dito anteriormente, $\mathrm{RN}$ possuem especificidades, tais como a imaturidade dos sistemas orgânicos, podendo levar a aumento do risco de eventos adversos relacionados ao uso de medicamentos ${ }^{(19)}$.

Pode-se afirmar que eventos adversos relacionados aos RN da Unidade Neonatal podem ser graves e até fatais. Geralmente, os RN da unidade estudada são prematuros, apresentam baixo peso e/ou estão em condições graves, são manipulados por diversos profissionais, permanecem por um longo tempo hospitalizado, e estão expostos a diversos procedimentos, aumentando a possibilidade de ocorrência de eventos adversos.

Foram observadas poucas anotações sobre a necessidade de troca da pulseira de identificação; e em relação aos registros presentes em prontuário, em 81,5\% dos casos não havia informação sobre a colocação da pulseira. Informações sobre localização, legibilidade, integridade ou necessidade de troca também foram poucas.

Assim como foi verificada a ausência da pulseira e seu uso incorreto durante a internação, também foram observados poucos registros referentes à sua presença, demonstrando que os profissionais de saúde investigados podem não ter sido orientados ou não estarem sensíveis a essa prática de segurança.

Alguns registros encontrados eram provenientes de outras unidades, como o Centro Obstétrico e o Alojamento Conjunto, que parecem ter outra percepção do uso da pulseira de identificação. Além disso, no Alojamento Conjunto, o registro diário da presença da pulseira de identificação faz parte de uma rotina e está inserido na Sistematização da Assistência de Enfermagem (SAE), preconizada pela Resolução do Conselho Federal de Enfermagem n 358 de 15 de outubro de $2009^{(20)}$.

O estabelecimento de rotinas institucionais possivelmente melhora a adesão ao uso das pulseiras. Com relação à anotação em prontuário, é importante que os profissionais sejam orientados e sensibilizados quanto à relevância assistencial, administrativa e documental de se registrar a colocação, legibilidade, integridade, e localização da pulseira.

Dos itens citados nos registros em prontuários, a localização da pulseira apresentou maior frequência e não foram observadas anotações sobre a presença das duas pulseiras de identificação, conforme orienta a rotina institucional. Há de se destacar que no Brasil, poucos estudos foram publicados a respeito da identificação do paciente pediátrico e da conferência desta pelo profissional de saúde antes da realização dos procedimentos ${ }^{(3)}$.

Entende-se como limitação do estudo a diminuição de internações pediátricas, devido a redução do número total de leitos na instituição onde foi realizada a pesquisa.

\section{CONCLUSÃO}

Verificou-se que os profissionais da unidade investigada apresentam dificuldades quanto à correta identificação do paciente. Existe urgente necessidade de maior orientação e envolvimento dos profissionais de enfermagem quanto à importância da adequada identificação do neonato, implicando o uso de estratégias facilitadoras desse processo.

Percebeu-se que o estabelecimento de rotinas institucionais inseridas na SAE, com foco na correta 
identificação do RN através de pulseira, melhora a prática assistencial, e apesar da existência de um protocolo de identificação do paciente, os profissionais não se apropriaram deste.

Os resultados demonstram que é fundamental intensificar o processo de educação continuada, no que diz respeito à identificação correta do paciente, especialmente na Unidade Neonatal, em que o risco de erros é grande, e os eventos adversos potencialmente graves. Identificar o paciente por meio do uso de pulseira corresponde a um processo eficaz, de baixo custo e fácil implementação.

Ficou evidenciada a complexidade do processo de identificação do paciente neonatal, que vai muito além do ato de fixar a pulseira de identificação após o nascimento. A identificação compreende a checagem diária da presença e integridade da pulseira, legibilidade dos descritores, e checagem sistemática da pulseira antes da realização de qualquer procedimento.

Torna-se necessário e urgente fomentar a cultura de segurança nas instituições hospitalares, envolvendo toda a equipe de saúde que atua junto ao neonato, visando à prevenção de eventos adversos. Quanto melhores forem definidos os fluxos relacionados a esse processo e quanto mais os profissionais estiverem envolvidos com a identificação do $\mathrm{RN}$, melhor e mais segura será a assistência em saúde prestada a estes pacientes.

\section{REFERÊNCIAS}

1. de Souza FT, Garcia MC, Rangel PPS, Rocha PK. Percepção da enfermagem sobre os fatores de risco que envolvem a segurança do paciente pediátrico. Rev Enferm UFSM. 2014;4(1):152-62.

2. Tase TH, Lourenção DCA, Bianchini SM, Tronchin DMR. Identificação do paciente nas organizações de saúde: uma reflexão emergente. Rev. Gaúcha Enferm. [Internet] 2013;34(3) [acesso em 02 jun 2016]. Disponível: http:// dx.doi.org/10.1590/S1983-14472013000300025.

3. Porto TP, Rocha PK, Lessmann JC, Souza S, Kretzer L, Anders JC. Identificação do paciente em unidade pediátrica: Uma questão de segurança. Rev. Soc. Bras. Enferm. Ped. [Internet] 2011;11(2) [acesso em 20 mar 2016]. Disponível: http://www.sobep.org.br/revista/images/stories/pdf-revista/vol11-n2/v.11_n.2-art1.a-identificacaodo-paciente.pdf.

4. Lanzillotti LS, de Seta MH, de Andrade CLT, Mendes Junior WV. Eventos adversos e outros incidentes na unidade de terapia intensiva neonatal. Ciênc. saúde coletiva. [Internet] 2015;20(3) [acesso em 20 mar 2016]. Disponível: http://dx.doi.org/10.1590/1413-81232015203.16912013.

5. Brasil. Ministério da Saúde. Portaria $n^{\circ} 529$, de $1^{\circ}$ de abril de 2013. Institui o Programa Nacional de Segurança do Paciente. Diário Oficial da União, Brasília, 02 abr. 2013. Seção 1. Pag. 43.

6. Medronho RA. Epidemiologia. $2^{\text {a }}$ ed. São Paulo: Atheneu; 2008.

7. Kauark F, Manhães FC, Medeirios CH. Metodologia da pesquisa: Um guia prático. Itabuna: Via Litterarum; 2010.

8. Marconi MA, Lakatos EM. Fundamentos de Metodologia Científica. $5^{a}$ ed. São Paulo: Atlas; 2003.

9. Ministério da Saúde (BR). Protocolo de identificação do paciente. Brasília: Ministério da Saúde; 2013.

10. Ministério da Saúde (BR). Conselho Nacional de Saúde. Diretrizes e normas regulamentadoras de pesquisa envolvendo seres humanos. Resolução n. 466, de 12 de dezembro de 2012. Brasília (DF); 2012.

11. Joint Commission International. Metas Internacionais de Segurança do Paciente. [Internet] 2015 [acesso em 11 out 2016]. Disponível: http://www.jointcommissioninternational.org/improve/international-patient-safety-goals/.

12. Conselho Regional de Enfermagem do Estado de São Paulo (COREN-SP). Rede Brasileira de Enfermagem e Segurança do Paciente (REBRAENSP). 10 passos para a segurança do paciente. [Internet] São Paulo: COREN-SP/ REBRAENSP; 2010 [acesso em 11 out 2016]. Disponível: http://www.coren-sp.gov.br/sites/default/files/10_passos_ seguranca_paciente_0.pdf.

13. World Health Organization (WHO). Joint Commission Resources. Joint Commission International. Patient 
Safety Solutions. Solution 2: patient identification [Internet]. Geneva; 2007;1 [acesso em 11 out 2016]. Disponível: http://www.who.int/patientsafety/solutions/patientsafety/PS-Solution2.pdf.

14. Quadrado ERS, Tronchin DMR. Avaliação do protocolo de identificação do neonato de um hospital privado. Rev. Latino-Am. Enfermagem. [Internet] 2012; 20(4) [acesso em 16 mar 2016]. Disponível: http://dx.doi.org/10.1590/ S0104-11692012000400005.

15. Agência Nacional de Vigilância Sanitária (BR). Assistência segura: uma reflexão teórica aplicada à prática. [Internet] 2013 [acesso em 18 fev 2016]. Disponível: http://www20.anvisa.gov.br/segurancadopaciente/images/ documentos/livros/Livro1-Assistencia_Segura.pdf.

16. Tomazoni A. Cultura de segurança do paciente em unidades de terapia intensiva neonatal. Santa Catarina [dissertação]. Santa Catarina: Universidade Federal de Santa Catarina; 2013.

17. Ventura CMU, Alvez JGB, Meneses JA. Eventos adversos em Unidade de Terapia Intensiva Neonatal. Rev. bras. enferm. 2012;65(1):49-55.

18. Teixeira TCA, Cassiani SHB. Análise de causa raiz: avaliação de erros de medicação em um Hospital Universitário. Rev. esc. enferm. USP. 2010;44(1):139-46.

19. Leitão ALP. Notificação de incidentes e eventos adversos em neonatologia [dissertação]. Lisboa (Portugal): Universidade Nova de Lisboa. Escola Nacional de Saúde Pública; 2011.

20. Conselho Federal de Enfermagem. Resolução n. 358, de 15 de outubro de 2009. Dispõe sobre a Sistematização da Assistência de Enfermagem e a implementação do Processo de Enfermagem em ambientes, públicos ou privados, em que ocorre o cuidado profissional de Enfermagem, e dá outras providências. Rio de Janeiro: COFEN; 2009. 